\title{
La fibromialgia vista por los Médicos especialistas en medicina de dolor en Colombia
}

Publicado:

6 de Noviembre, 2020 
Daniel G. Fernández-Ávila, MD. MSc. PhD.

Hospital Universitario San Ignacio -

Pontificia Universidad Javeriana,

Bogotá, Colombia

daniel.fernandez@javeriana.edu.co

ORCID: 0000-0003-1490-1822

Diana María Ronderos-Botero, MD

Internal Medicine Service, BronxCare Hospital

Center, New York, USA

dianamariaronderos@gmail.com

ORCID: 0000-0001-4581-5643

Diana N. Rincón-Riaño, MD

Reumatóloga, Artmédica.

Medellín, Colombia

dncolombia@hotmail.com

ORCID: 0000-0001-6182-4036

Juan M. Gutiérrez, MD

Reumatólogo, Hospital Universitario San

Ignacio - Pontificia Universidad Javeriana.

Bogotá, Colombia

jmgutierrez47@hotmail.com

ORCID: 0000-0002-8549-661X 
ARTíCULO CIENTÍFICO

La Fibromialgia vista por los

Médicos Especialistas en medicina

de dolor en Colombia 


\section{en Español}

\section{Introducción}

Los conceptos y las percepciones sobre el diagnóstico y el tratamiento de la fibromialgia, entre los médicos especialistas en medicina del dolor no se conocen objetivamente, lo cual nos motivo a llevar a cabo el presente estudio.

\section{Método}

Estudio descriptivo de corte transversal. A través de un grupo focal en el que participaron dos reumatólogos, dos médicos especialistas en medicina del dolor, y un experto en métodos de investigación cualitativa, se diseñó una encuesta para evaluar las percepciones y conceptos que los médicos especialistas en medicina del dolor tienen sobre el diagnóstico y el tratamiento de la fibromialgia. La encuesta se aplicó de forma autoadministrada y anónima a través de un link en internet, el cual fue enviado a médicos especialistas en dolor, pertenecientes a la Asociación Colombiana Para el Estudio del dolor.

\section{Resultados}

Encuesta aplicada a 81 médicos especialistas en medicina del dolor. 71.6\% $(n=58)$ considera que hay suficiente evidencia para considerar la fibromialgia como una enfermedad, 90.1\% ( $n=73)$ usa los criterios ACR de 1990 para diagnosticar pacientes con fibromialgia y $60.5 \%(n=49)$ de los encuestados manifestó emplear los criterios de clasificación de 2010. Los medicamentos más formulados para manejo de fibromialgia son los antidepresivos (96.3\%) seguido de anticonvulsivantes (88.9\%) y analgésicos (84\%). $84 \%$ de los médicos remite estos pacientes a psiquiatría y 63\% al reumatólogo.

\section{Conclusión}

El presente estudio muestra información acerca de las percepciones acerca del diagnóstico y tratamiento de fibromialgia entre un grupo de médicos especialistas en medicina del dolor colombianos. 


\section{en Inglés}

\section{Introduction}

The concepts and perceptions about the diagnosis and treatment of fibromyalgia are not objectively known among doctors specialized in pain medicine, which motivated us to carry out this study.

\section{Methods}

Descriptive cross-sectional study. Through a focus group in which two rheumatologists, two pain medicine specialists, and an expert in qualitative research methods participated, a survey was designed to evaluate the perceptions and concepts that pain medicine specialists have about the diagnosis and treatment of fibromyalgia. The survey was applied in a self-administered and anonymous way through an internet link, which was sent to pain specialists, belonging to the Colombian Association for the Study of pain.

\section{Results}

Survey applied to 81 doctors specialized in pain medicine. $71.6 \%(n=58)$ consider that there is sufficient evidence to consider fibromyalgia as a disease, $90.1 \%(n=73)$ use the 1990 ACR criteria to diagnose fibromyalgia patients and $60.5 \%$ ( $n=$ 49) of the respondents stated use the 2010 classification criteria. The most formulated medications for fibromyalgia management are antidepressants (96.3\%) followed by anticonvulsants (88.9\%) and analgesics (84\%). $84 \%$ of doctors refer these patients to psychiatry and $63 \%$ to a rheumatologist.

\section{Conclusion}

The present study shows information about the perceptions about the diagnosis and treatment of fibromyalgia among a group of Colombian pain medicine doctors 


\section{en Portugués}

\section{Introdução}

Os conceitos e percepções sobre o diagnóstico e tratamento da fibromialgia não são objetivamente conhecidos entre os médicos especialistas em medicina da dor, o que nos motivou a realizar este estudo.

\section{Métodos}

Estudo descritivo transversal. Através de um grupo focal no qual dois reumatologistas, dois especialistas em medicina da dor e um especialista em métodos de pesquisa qualitativa participaram, uma pesquisa foi projetada para avaliar as percepções e conceitos que os especialistas em medicina da dor têm sobre o diagnóstico e tratamento da fibromialgia. A pesquisa foi aplicada de forma autoadministrada e anônima por meio de um link da Internet, enviado a especialistas em dor, pertencentes à Associação Colombiana para o Estudo da Dor.

\section{Resultados}

Pesquisa aplicada a 81 médicos especializados em medicina da dor. $71,6 \%(n=58)$ consideram que existem evidências suficientes para considerar a fibromialgia como uma doença, 90,1\% ( $n=73$ ) usam os critérios da ACR de 1990 para diagnosticar pacientes com fibromialgia e $60,5 \%$ ( $n=49)$ dos entrevistados declararam use os critérios de classificação de 2010. Os medicamentos mais formulados para o tratamento da fibromialgia são antidepressivos (96,3\%), seguidos de anticonvulsivantes $(88,9 \%)$ e analgésicos (84\%). 84\% dos médicos encaminham esses pacientes para psiquiatria e $63 \%$ para um reumatologista.

\section{Conclusão}

O presente estudo mostra informações sobre as percepções sobre o diagnóstico e tratamento da fibromialgia em um grupo de médicos colombianos de analgésicos 
La fibromialgia es una enfermedad crónica cuyo síntoma característico es el dolor músculo esquelético generalizado, usualmente asociado con una variada gama de síntomas que, junto con el dolor, alteran la calidad de vida de los pacientes (1).

Fue reconocida como enfermedad por la Organización Mundial de la Salud (OMS) en 1992, como un síndrome clínico de etiología desconocida que provoca dolor musculoesquelético crónico, difuso e incapacitante, y suele acompañarse de trastornos como fatiga, alteraciones del sueño, cefalea, colon irritable, entre otros (2).

La prevalencia de la fibromialgia varia entre $2 \%$ a $4 \%$ de la población general, con un mayor compromiso en mujeres, (relación mujer : hombre de $9: 1)(3,4)$.

En Colombia se estima una prevalencia de 0,72\% (IC 95\%: 0,47-1,11\%), la cual se determinó en un estudio que utilizó la metodología y cuestionario de tamización COPCORD (Community Oriented Program in the Rheumatic Diseases), tras evaluar 6.693 personas de 6 ciudades de Colombia (5). Los criterios de clasificación de la fibromialgia fueron definidos inicialmente en 1990 por parte del Colegio Americano de Reumatología (ACR) (6).

Durante los siguientes 20 años se hicieron varias observaciones por parte de diferentes autores, incluyendo al autor principal de los criterios de 1990, para buscar una nueva forma de clasificar a este tipo de pacientes (7).

En mayo de 2010 se propusieron nuevos criterios de clasificación (8), buscando incluir no solo aspectos relacionados con el dolor, sino con el complejo espectro de síntomas que afectan a estos pacientes. La fibromialgia representa un reto diagnóstico y terapéutico para el médico, independientemente de la especialidad que este tenga, dada la complejidad que posee una enfermedad caracterizada por la presencia de 
dolor generalizado como principal síntoma, asociado con otros hallazgos clínicos difíciles de cuantificar como fatiga, trastornos del sueño y alteraciones cognitivas (9).

Varios de estos síntomas pueden estar relacionados con otras enfermedades osteomusculares, especialmente durante las primeras etapas de una enfermedad, lo cual dificulta el proceso diagnóstico (10).

Los pacientes con fibromialgia generalmente asisten a múltiples médicos especialistas, tanto al inicio de síntomas para confirmar el diagnóstico, como durante el tiempo de tratamiento y seguimiento clínico (11).

El médico especialista en medicina del dolor juega un papel clave en el abordaje y tratamiento de un dolor crónico sin causa subyacente aparente, y el enfoque de síndrome de "dolor neuropático" o "no nociceptivo" o "sensibilidad central", donde la hiperalgesia y alodinia son típicas $(12,13)$.

Los conceptos sobre el diagnóstico y el tratamiento de fibromialgia entre médicos especialistas en medicina del dolor de Colombia no se conocen objetivamente, hasta ahora solo se dispone de información de carácter informal y subjetiva no publicada. El objetivo de este estudio es obtener datos objetivos sobre este tema, y describir los conceptos y percepciones sobre el diagnóstico y tratamiento de la fibromialgia entre los médicos especialistas en medicina del dolor colombianos. 
Estudio descriptivo de corte transversal. Mediante un grupo focal conformado por dos médicos reumatólogos, un médico especialista en medicina del dolor y un experto en métodos de investigación cualitativa, se diseñó una encuesta que estaba conformada por cuatro dominios. El primer dominio sobre aspectos generales y de identificación del médico (edad, género, tiempo de experiencia y ciudad donde ejerce la profesión); el segundo dominio interrogaba sobre los aspectos diagnósticos de la enfermedad; el tercero sobre las opciones terapéuticas utilizadas (tratamientos farmacológicos, no farmacológicos y terapias alternativas); el cuarto dominio examina la opinión sobre el manejo multidisciplinario de la fibromialgia y la derivación a otro especialista. Las opciones de respuesta se presentaron como una escala Likert. Se realizó una prueba piloto con 10 residentes de medicina del dolor para evaluar la duración de la encuesta y el nivel de comprensión de las preguntas. La encuesta se aplicó de forma autoadministrada y anónima a través de un link en internet, el cual fue enviado a médicos especialistas en dolor, pertenecientes a la Asociación Colombiana Para el Estudio del Dolor. La información se recopiló en una base de datos y se analizó en Microsoft Excel®. Se realizó un análisis descriptivo, utilizando frecuencias y porcentajes para las variables cualitativas y medidas de tendencia central para las variables cuantitativas. 
Result ados

La encuesta fue respondida por 81 médicos especialistas en medicina del dolor. $72.8 \%$ ( $n=$ 59) de los encuestados eran hombres, $49,4 \%$ ( $n=$ 40) eran mayores de 50 años. El $71.6 \%(n=58)$ de los especialistas considera que hay suficiente evidencia para considerar a la fibromialgia como una enfermedad. $48.1 \%(n=39)$ considera que los criterios ACR 1990 son suficientes para diagnosticar fibromialgia y mas del $90 \%$ de estos especialistas los usan para el enfoque diagnóstico de pacientes con sospecha de fibromialgia sin embargo, hasta un $60.5 \%$ ( $n=49)$ de los especialistas han utilizado también los criterios de clasificación del ACR de 2010. El 43.2 $\%(n=35)$ de los entrevistados considera que el paciente con fibromialgia se siente rechazado por su especialidad y el $82.7 \%(n=67)$ considera que los pacientes son rechazados por médicos de otras especialidades.

En cuanto a aspectos relacionados con el tratamiento de la enfermedad, se encontró que los medicamentos más empleados por los médicos especialistas en medicina del dolor en el tratamiento de la fibromialgia son los antidepresivos, prescritos por $96.3 \%$ de los encuestados, seguido de anticonvulsivantes (88.9 \%) y analgésicos (84\%).

En cuanto a los antidepresivos, el más empleado es amitriptilina (52.1\%), seguido por fluoxetina (48 \%) y duloxetina (39.1\%). En el grupo de los antiepilépticos el más utilizado es pregabalina (87.1 \%), seguido de gabapentín (79.3 \%) y carbamazepina (74.4\%). El analgésico mas frecuentemente formulado es el acetaminofén mas tramadol (57.7\%), seguido por acetaminofén mas tizanidina (54.2 \%), siendo los antiinflamatorios no esteroideos (AINES) de muy baja formulación. (Figura 1).

En cuanto al manejo no farmacológico, encontramos que $58 \%(n=47)$ de los médicos especialistas en dolor prescribe hidroterapia 
como parte del manejo del paciente con fibromialgia. En lo referente a remisión a otras especialidades para manejo interdisciplinario, se encontró que 84 \% $(n=68)$ de los especialistas remite a psiquiatría y 63 $\%(n=51)$ remiten a reumatología.

Finalmente el $48 \%$ de los especialistas entrevistados considera que el paciente con fibromialgia debe tener para su manejo un equipo multidisciplinario liderado por el médico especialista en medicina física y rehabilitación y solo el 28 \% considera que debe ser liderado por el especialista en medicina del dolor (Figura 2).

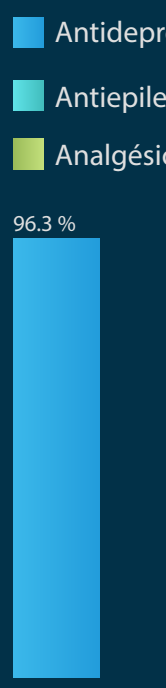

Antidepresivos

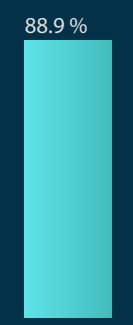

Antiepilepticos

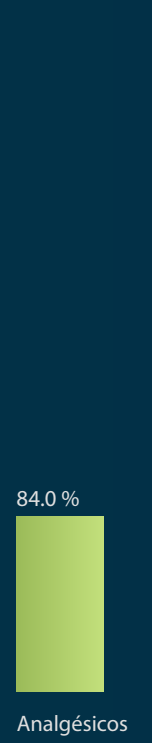

*Figura 1
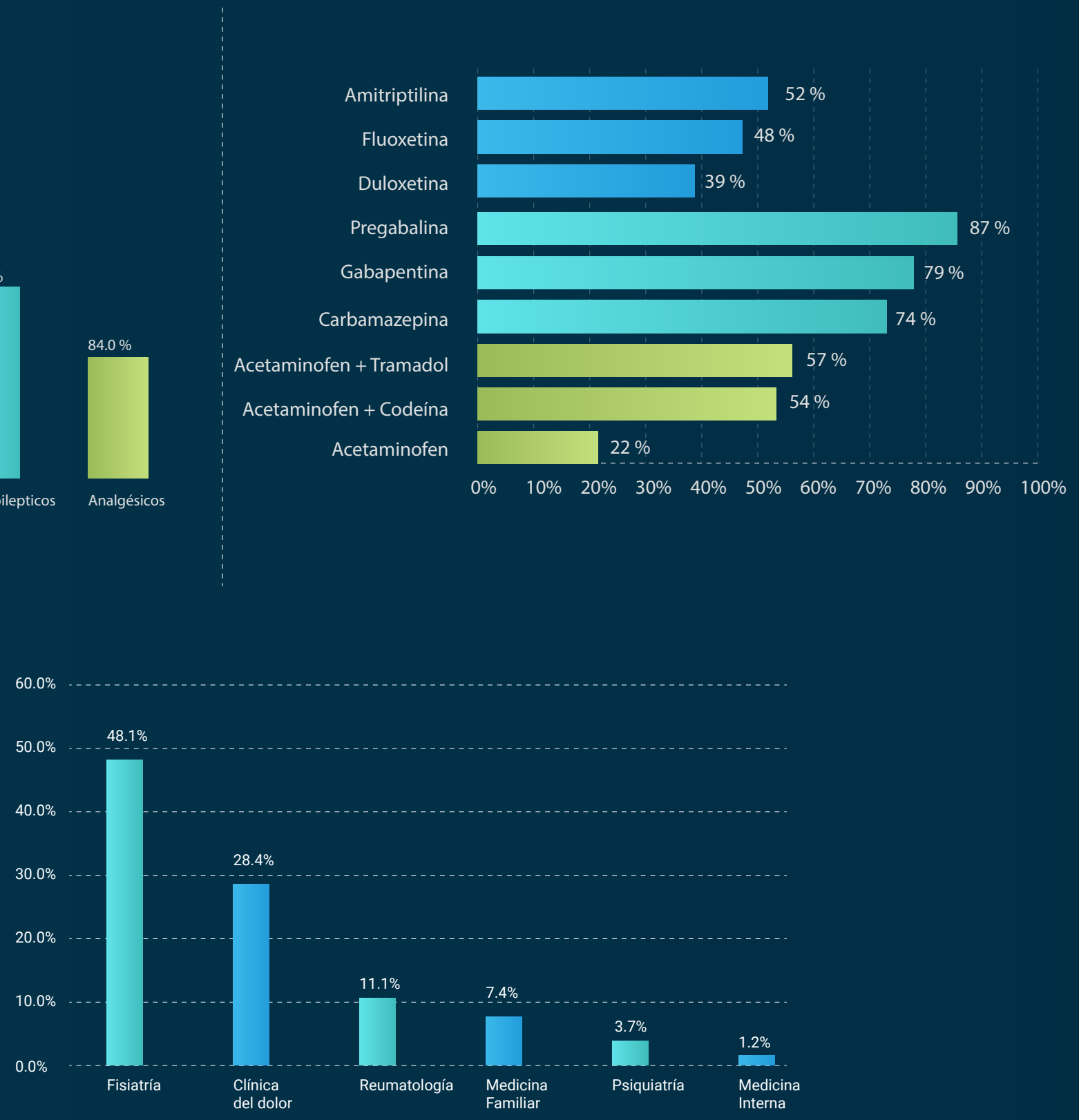

*Figura 2 


\section{Discusión y conclusiones}

\section{Discusión}

Nuestro estudio es el primero en describir los conceptos y percepciones de médicos especialistas en medicina del dolor, en torno su abordaje diagnóstico y terapéutico de pacientes con fibromialgia. El primer punto destacado que describimos es que siete de cada diez médicos especialistas en medicina del dolor, considera la fibromialgia como una enfermedad, a diferencia de otras especialidades donde se deslegitima la real existencia de esta condición $(14,15)$. Existen estudios donde se han expuesto las dificultades en el diagnóstico, abordaje y tratamiento de la fibromialgia en medicina general y otras especialidades como psiquiatría, medicina física y rehabilitación y reumatología, reportando que su entrenamiento en temas relacionados con fibromialgia es inadecuado (16-18). El tema que mayor preocupa a diferentes especialistas, es la 
insatisfacción con el retraso para llegar a un diagnóstico, generando muchas veces un sentimiento de frustración por no poder ayudar al paciente, debido a una gran incertidumbre en la etiología de esta enfermedad, demorando el manejo y obteniendo resultados muy variables tras el inicio del tratamiento, muchas veces insatisfactorios $(19,20)$. En cuanto al uso de criterios diagnósticos, los criterios del American Collegue of Rheumatology (ACR) de 1990, son usados por casi el $90 \%$ de los médicos especialistas en medicina del dolor para el abordaje diagnóstico de este tipo de pacientes, estos datos son similares a otras especialidades, como lo reporta el estudio de $\mathrm{Mu} \mathrm{R}$. et al., donde $83.7 \%$ de los reumatólogos utilizan aún criterios del ACR de 1990 (18). Sin embargo, los criterios diagnósticos de 2010 son usados por también por el $61 \%$ de los médicos especialistas en medicina del dolor, mucho mayor a lo reportado en otros estudios (21). Una investigación comparó el uso de criterios ACR de 1990 entre reumatólogos latinoamericanos y europeos, encontrando un porcentaje de uso del $61.7 \%$ y $35.7 \%$ respectivamente, datos similares en comparación con nuestros resultados (22).

En cuanto a aspectos del tratamiento de la enfermedad, encontramos que los médicos especialistas en medicina del dolor colombianos brindan, en general, un manejo médico acorde con guías internacionales de tratamiento $(1,23)$, empleando como principales tratamiento antidepresivos la amitriptilina, fluoxetina y duloxetina y de anticonvulsivantes la pregabalina y el gabapentín, y de analgésicos la preferencia por la asociación de acetaminofén mas opioide leve o con relajante muscular, por encima de acetaminofén solo, y el uso limitado de antiinflamatorios no esteroideos acertadamente, pues dichos medicamentos no son recomendados para el manejo de esta enfermedad.

Es clara la importancia que se le da al manejo interdisciplinario de esta entidad, con remisiones casi del $85 \%$ de los pacientes a especialidades como psiquiatría y $64 \%$ a reumatología, y considerando en mas del 50\% que el médico a la cabeza del manejo interdisciplinario es el médico de medicina física y rehabilitación.

Los pacientes con fibromialgia deben enfrentar no solo el cuadro de dolor y los síntomas asociados con el síndrome, sino también la falta de aceptación por parte de familiares (24), e incluso de médicos 
relacionados con su manejo. Es necesario considerar como real el dolor en los pacientes con fibromialgia, pues a pesar de los interrogantes lanzados desde diferentes especialidades médicas, hay evidencia suficiente para considerar la existencia de esta enfermedad, y es necesario profundizar en el conocimiento sobre su fisiopatología, diagnóstico y tratamiento $(25,26)$

El presente estudio describe información objetiva sobre aspectos claves de la fibromialgia, de la que solamente se disponía a través de datos totalmente subjetivos, que no iban más allá de comentarios informales entre especialistas. Esta información puede ser empleada como una retroalimentación a los mismos colegas, así como ser la base para otros estudios como, por ejemplo, la aplicación de esta misma encuesta a otras especialidades implicadas en el manejo de la fibromialgia como psiquiatría, medicina interna y medicina familiar, especialidades que también tiene contacto con esta enfermedad. Actualmente nuestro grupo de investigación avanza en la aplicación de esta encuesta a otros especialistas en Colombia y otros países de América Latina.

\section{Conclusión}

Se muestra información objetiva sobre las percepciones de la fibromialgia entre un grupo de médicos especialistas en medicina del dolor colombianos, información que será útil como retroalimentación a estos especialistas y como base de otros estudios que lleven a aumentar la cantidad y calidad de información respecto al mejor abordaje de .esta compleja entidad, 
1. Clauw DJ. Fibromyalgia: a clinical review. JAMA. 2014 Apr 16;311(15):1547-55.

2. Goldenberg DL. Diagnosis and Differential Diagnosis of Fibromyalgia. Am J Med. 2009 Dec;122(12 SUPPL.).

3. Vincent A, Lahr BD, Wolfe F, Clauw DJ, Whipple MO, Oh TH, et al. Prevalence of fibromyalgia: a population-based study in Olmsted County, Minnesota, utilizing the Rochester Epidemiology Project. Arthritis Care Res (Hoboken). 2013 May;65(5):786-92.

4. Wolfe F, Ross K, Anderson J, Russell IJ, Hebert $L$. The prevalence and characteristics of fibromyalgia in the general population. Arthritis Rheum. 1995;38(1):19-28.

5. Santos A, Rueda J, Angarita J-I, Giraldo R, Forero E, Pelaez-Ballestas $\mathrm{l}$, et al. FRI0694 Prevalence of rheumatic disease in an adult population from colombia. a copcord methodology study. In: Poster Presentations. BMJ Publishing Group Ltd and European League Against Rheumatism; 2017. p. 752.3-753.

6. Wolfe F, Smythe HA, Yunus MB et al. The American College of Rheumatology 1990 Criteria for the Classification of Fibromyalgia: report of the Multicenter Criteria Committee. Arthritis
Rheum. 1990;33:60-172.

7. Wolfe F. Stop using the American College of Rheumatology criteria in the clinic. Journal of Rheumatology. 2003 Aug 1;30(8):1671-2.

8. Wolfe F, Clauw DJ, Fitzcharles MA, Goldenberg $D L$, Katz RS, Mease P, et al. The American College of Rheumatology preliminary diagnostic criteria for fibromyalgia and measurement of symptom severity. Arthritis Care Res. 2010;62(5):600-10.

9. Chakrabarty S, Zoorob R. Fibromyalgia. Am Fam Physician. 2007;15(76(2)):247-54.

10. Häuser W, Sarzi-Puttini P, Fitzcharles MA. Fibromyalgia syndrome: under-, over- and misdiagnosis. Clin Exp Rheumatol. 2019;37(1):90-7.

11. Busse JW, Ebrahim S, Connell G, Coomes EA, Bruno P, Malik K, et al. Systematic review and network meta-analysis of interventions for fibromyalgia: a protocol. Syst Rev. 2013 Mar 13;2:18.

12. Dadabhoy D, Clauw DJ. Therapy Insight: fibromyalgia--a different type of pain needing a different type of treatment. Nat Clin Pract Rheumatol. 2006 Jul;2(7):364-72. 
13. Cassisi G, Sarzi-Puttini P, Casale R, Cazzola M, Boccassini $L$, Atzeni $F$, et al. Pain in fibromyalgia and related conditions. Vol. 66, Reumatismo. Page Press Publications; 2014. p. 72-86.

14. Zavestoski S, Brown P, McCormick S, Mayer B, D'Ottavi M, Lucove JC. Patient activism and the struggle for diagnosis: Gulf War illnesses and other medically unexplained physical symptoms in the US. Soc Sci Med. 2004 Jan;58(1):161-75.

15. Ware NC. Suffering and the Social Construction of Illness: The Delegitimation of Illness Experience in Chronic Fatigue Syndrome. Vol. 6, Medical Anthropology Quarterly. 1992;6:347-61.

16. Perrot S, Choy E, Petersel D, Ginovker A, Kramer E. Survey of physician experiences and perceptions about the diagnosis and treatment of fibromyalgia. BMC Health Serv Res. 2012;12(1):1.

17. Blotman F, Thomas E, Myon E, Andre E, Caubere JP, Taiieb C. Awareness and knowledge of fibromyalgia among French rheumatologists and general practitioners. Clin Exp Rheumatol. 2005;23(5):697-700.

18. Mu R, Li C, Zhu JX, Zhang XY, Duan TJ, Feng M, et al. National survey of knowledge, attitude and practice of fibromyalgia among rheumatologists in China. Int J Rheum Dis. 2013;16(3):258-63.

19. Briones-Vozmediano E, Vives-Cases C, Ronda-Pérez E, Gil-González D. Patients' and professionals' views on managing fibromyalgia. Pain Res Manag. 2013;18(1):19-24.

20. Ubago Linares M del C, Ruiz I, Bermejo MJ, Olry de Labry Lima A, Plazaola Castaño J.
Características clínicas y psicosociales de personas con fibromialgia. Repercusión del diagnóstico sobre sus actividades. Rev Esp Salud Publica. 2005;79:683-95.

21. Bloom S, Ablin JN, Lebel D, Rath E, Faran $Y$, Daphna-Tekoah $S$, et al. Awareness of diagnostic and clinical features of fibromyalgia among orthopedic surgeons. Rheumatol Int. 2013 Apr;33(4):927-31.

22. Clark P, Paiva ES, Ginovker A, Salomón PA. A patient and physician survey of fibromyalgia across Latin America and Europe. BMC Musculoskelet Disord. 2013 Jun 14;14:188.

23. Macfarlane GJ, Kronisch C, Dean LE, Atzeni F, Häuser W, Flub E, et al. EULAR revised recommendations for the management of fibromyalgia. Ann Rheum Dis. 2017 Feb $1 ; 76(2): 318-28$.

24. Kool MB, Van Middendorp H, Boeije HR, Geenen R. Understanding the lack of understanding: Invalidation from the perspective of the patient with fibromyalgia. Arthritis Care Res. 2009 Dec 15;61(12):1650-6.

25. Hadker N, Garg S, Chandran AB, Crean SM, McNett MM, Silverman SL. Efficient practices associated with diagnosis, treatment and management of fibromyalgia among primary care physicians. Pain Res Manag. 16(6):440-4.

26. Coskun Benlidayi I. Fibromyalgia as a challenge for patients and physicians. Rheumatol Int. 2018 Dec 1;38(12):2345. 
GLOBAL RHEUMATOLOGY 